\title{
CONVEX SETS AND NEAREST POINTS ${ }^{1}$
}

\section{R. R. PHELPS ${ }^{2}$}

1. Introduction. A well known theorem due to Motzkin [11] and extended by Busemann [3] and Jessen [7] characterizes the convexity of a closed set $S$ in Euclidean $n$-space $E^{n}$ in the following manner: $S$ is convex if and only if to each point in $E^{n}$ there corresponds a unique nearest point in $S$. We show here that if $S$ is convex, the set $S_{z}$ of all points having $z$ as a nearest point in $S$ is a convex cone with vertex $z$, while the hypothesis that $S_{z}$ be merely a cone with vertex $z$ (for each $z \in S$ ) is shown to characterize the convexity of $S$.

In trying to establish these results in a more general normed linear space $E$ we find that the statement " $S_{z}$ is convex whenever $S$ is convex" is equivalent to the existence of an inner product in $E$ when the dimension of $E$ is at least three, while in a two-dimensional space it is equivalent to strict convexity. A theorem by Motzkin [12] leads easily to the analogous result that $E$ is an inner product space if and only if $S_{z}$ is convex for every $S \subset E$ (and $z \in S$ ).

In the concluding section we consider a nearest-point map $f$ which assigns to each point of $E$ a nearest point in a given closed set $S$. It is shown that the property " $f$ shrinks distances whenever it exists for a closed convex set" characterizes inner product spaces of three or more dimensions. In two-dimensional spaces this property is equivalent to strict convexity and symmetry of Birkhoff's orthogonality [2]. The convexity of a closed set in $E^{n}$ is shown to be characterized by the fact that its nearest-point map shrinks distances.

2. Definitions and remarks. Throughout this paper $E$ will be a normed linear space and $S$ a subset thereof. For $z \in S, S_{z}$ will be the set $\left\{x:\|x-z\|=\inf _{y \in S}\|x-y\|\right\}$, the set of all points in $E$ having $z$ as a nearest point in $S$. It may well be that $z$ is the only point in $S_{z}$. It is not difficult to verify that if a sequence of points in $S_{z}$ converges then its limit is in $S_{z}$; hence $S_{z}$ is always closed.

We will say that $S$ is proximinal ${ }^{3}$ if for each point $x$ in $E$ there is a

Presented to the Society, August 24, 1956; received by the editors July 20, 1956 and, in revised form, December 8, 1956.

${ }^{1}$ Work on this paper sponsored in part by the Office of Ordnance Research, U. S. Army, under contract DA-04-200-ORD-292.

2 The author wishes to thank Professor V. L. Klee for many valuable suggestions and criticisms.

${ }^{3}$ This word, a combination of proximity and minimal, was suggested by Raymond Killgrove. 
point of $S$ nearest to $x$, i.e., if for each point $x \in E$ there is at least one point $z$ in $S$ such that $x$ is in $S_{z}$. If there is a unique such $z$ for each $x$ in $E$ we will say that $S$ is uniquely proximinal. Although we are not primarily interested in conditions which guarantee that $S$ is proximinal we list some of the known ones:

(i) $S$ is proximinal if it is compact.

(ii) Every closed set $S$ is proximinal if $E$ is finite dimensional.

(iii) Every closed convex set $S$ is proximinal if $E$ is reflexive.

3. Cones, smoothness and strict convexity. The following lemma has appeared before [10] but will be proved here since we use it several times.

Lemma 3.1. If $S$ is convex and $z \in S$ then $S_{z}$ is a cone with vertex $z$.

Proof. We can suppose $z=\phi$. It will suffice to show that if $y \in S_{\phi}$, then $\lambda y \in S_{\phi}$ for each $\lambda>0$. Suppose $x \in S$, then $\|y\| \leqq\|y-x\|$. If $\lambda<1$, $\|\lambda y\|+\|y-\lambda y\|=\|y\| \leqq\|y-x\| \leqq\|y-\lambda y\|+\|\lambda y-x\|$, whence $\|\lambda y\|$ $\leqq\|\lambda y-x\|$. If $\lambda>1, \lambda^{-1} x$ is in the convex set $S$ so $\|\lambda y\|=\lambda\|y\|$ $\leqq \lambda\left\|y-\lambda^{-1} x\right\|=\|\lambda y-x\|$. Hence $\lambda y \in S_{\phi}$ for each $\lambda>0$.

A set $E$ is strictly convex if the boundary of its unit cell contains no line segment, i.e., if $\|x\|=1=\|y\|$ and $\lambda \in] 0,1[$ imply $\|\lambda x+(1-\lambda) y\|$ $<1$. The following lemma characterizes strict convexity in terms useful to us.

LEMMA 3.2. The following statements are equivalent:

(i) $E$ is strictly convex.

(ii) For each convex set $S$ and distinct points $x$ and $y$ of $S, S_{x} \cap S_{y}$ is empty.

(iii) Whenever a convex set $S$ is proximinal it is uniquely proximinal.

PROOF. (i) $\Rightarrow$ (ii). If $S_{x} \cap S_{y}$ is nonempty we can suppose $\phi \in S_{x} \cap S_{y}$, so $\|x\|=\|y\|$. Now, $(1 / 2)(x+y) \in S$ and if $E$ is strictly convex $\|(1 / 2)(x+y)\|<\|x\|=\|y\|$, a contradiction.

(ii) $\Rightarrow$ (iii). This is immediate from the definitions.

(iii) $\Rightarrow$ (i). Suppose $E$ is not strictly convex, then there exist distinct points $x$ and $y$ such that $\|\lambda x+(1-\lambda) y\|=1$ for each $\lambda \in[0,1]$. The (compact) convex line segment $[x, y]$ is proximinal but not uniquely proximinal since the origin is equidistant from all points of $[x, y]$.

We say that $E$ is smooth if its unit cell has a unique supporting hyperplane at each of its boundary points. (A hyperplane is a maximal proper closed linear variety.) We give a partial converse to Lemma 3.1 in the following lemma (stated but not proved in [10]).

Lemma 3.3. Suppose $S$ is closed and proximinal and $E$ is smooth. Then $S$ is convex if for each $z \in S, S_{z}$ is a cone with vertex $z$. 
Proof. Suppose $S$ is not convex. Then there exist points $u$ and $v$ in $S$ such that $] u, v[\subset E \sim S$. Let $x=(1 / 2)(u+v)$. Since $S$ is proximinal $x \in S_{z}$ for some $z \in S$ and we can suppose without loss of generality that $z=\phi$. Let $H$ be the unique hyperplane supporting $N_{\|x\|} \equiv\{y:\|y-x\|<\|x\|\}$ at $\phi$ and let $H^{\prime}$ be the open halfspace determined by $H$ which contains $N_{\|x\|} x$. Not both $u$ and $v$ are in the convex set $E \sim H^{\prime}$ since $x$ is not. Suppose $u \in H^{\prime}$. We will show that for some $\lambda>0, u \in N_{\|\lambda x\|} \lambda x=\lambda N_{\sharp x \|} x$ so that $\|\lambda x\|>\|\lambda x-u\|$ and hence $\lambda x \notin S_{\phi}$, contradicting the assumption that $S_{\phi}$ is a cone with vertex $\phi$.

Suppose that for every $\lambda>0, u \notin \lambda N_{\|x\|} x$. Then $\alpha u \bigoplus_{\|x\|} x$ for each $\alpha \in] 0,1\left[\right.$ and hence the convex set $\left[u, \phi\left[\right.\right.$ is disjoint from $N_{\|x\|} x$. There exists a hyperplane $G$ separating $\left[u, \phi\left[\right.\right.$ from $N_{\|x\|} x$ which necessarily supports $N_{\|x\|} x$ at $\phi$. But $u$ is in the closed halfspace determined by $G$ which does not contain $N_{\|x\|} x$ and hence $G \neq H$, contradicting the fact that $E$ is smooth. Thus for some $\lambda>0, u \in \lambda N_{\|x\|} x$, which was to be shown.

The assumption of smoothness of $E$ in Lemma 3.3 is a necessary one, since it is not difficult to show that the statement of the lemma, with smoothness omitted, implies that $E$ is smooth.

Since every closed subset of (smooth) $E^{n}$ is proximinal, Lemmas 3.1 and 3.3 combine to prove the following characterization of convexity.

TheOREM 3.4. A closed set $S$ in $E^{n}$ is convex if and only if for each $z \in S, S_{z}$ is a cone with vertex $z$.

4. Convex cones and inner products. We say that $E$ is an inner product space if $E$ admits an inner product $(x, y)$ such that $\|x\|$ $=(x, x)^{1 / 2}$. If $E$ is an inner product space it is easily verified that for points $x, y$ and $z$ in $E$ and $\lambda \in R$ the following identity holds:

$$
\begin{aligned}
& \|z-[\lambda x+(1-\lambda) y]\|^{2} \\
& \quad=\lambda\|x-z\|^{2}-\lambda(1-\lambda)\|x-y\|^{2}+(1-\lambda)\|z-y\|^{2} .
\end{aligned}
$$

(In fact [8] $E$ admits an inner product if and only if (1) holds true.)

In particular, we can see that an inner product space is strictly convex by setting $z=\phi,\|x\|=1=\|y\|$ and $\lambda \in] 0,1[$ in (1).

LeMma 4.1. If $E$ is an inner product space and $z \in S$ then $S_{z}$ is convex.

Proof. Suppose $z=\phi$ and that $x \in S_{\phi}$ and $y \in S_{\phi}$. Let $w$ $=\lambda x+(1-\lambda) y, \lambda \in] 0,1[$. Then if $v$ is any point of $S,\|x\| \leqq\|x-v\|$ and $\|y\| \leqq\|y-v\|$ while, by (1), we have

$$
\begin{aligned}
\|w\|^{2} & =\lambda\|x\|^{2}-\lambda(1-\lambda)\|x-y\|^{2}+(1-\lambda)\|y\|^{2} \\
& \leqq \lambda\|x-v\|^{2}-\lambda(1-\lambda)\|x-y\|^{2}+(1-\lambda)\|v-y\|^{2}=\|w-v\|^{2},
\end{aligned}
$$


so $\|w\| \leqq\|w-v\|$ and hence $w \in S_{\phi}$.

Motzkin [12] has proved the following interesting result: Suppose that $E$ is two-dimensional. Then $E$ is an inner product space if and only if for each set $S$ and $z \in S, S_{z}$ is convex. Since a normed linear space is an inner product space if and only if each two-dimensional subspace has an inner product [8], Motzkin's result leads easily to the sufficiency portion of the following theorem.

Theorem 4.2. A normed linear space $E$ is an inner product space if and only if for each set $S \subset E$ and $z \in S, S_{z}$ is convex.

Demanding convexity of $S_{z}$ only when $S$ itself is convex leads to the following result, closely related to Theorem 4.2 .

TheоRem 4.3. Suppose that the dimension of $E$ is at least three [resp. equal to two]. Then $E$ is an inner product space [resp. strictly convex] if and only if for each convex set $S$ and $z \in S, S_{z}$ is convex.

The portions of Theorem 4.3 which are as yet unproved follow from the next three lemmas. The idea used in the proof of the following lemma is due to James [6, Theorem 2].

Lemma 4.4. Suppose the dimension of $E$ is at least three. Then $E$ is an inner product space provided $S_{z}$ is convex for each convex set $S \subset E$ and $z \in S$.

Proof. If $x_{1}$ and $x_{2}$ are any two linearly independent points of $E$ there exist hyperplanes $H^{1}$ and $H^{2}$ such that $x_{1} \in H_{\phi}^{1}$ and $x_{2} \in H_{\phi}^{2}\left(H^{i}\right.$ $=h_{i}^{-1}(0)$, where $h_{i}$ is a continuous linear functional such that $h_{i}\left(x_{i}\right)$ $=\left\|x_{i}\right\|$ and $\left\|h_{i}\right\|=1\left[1\right.$, p. 55]). By Lemma $3.1, H_{\phi}^{1}$ and $H_{\phi}^{2}$ are convex cones with vertex $\phi$ and hence $\alpha_{i} x_{i} \in H_{\phi}^{i}$ if $\alpha_{i} \geqq 0(i=1,2)$. But $y \in H^{1}$ if and only if $-y \in H^{1}$, so $\left\|\left(-x_{1}\right)-y\right\|=\left\|x_{1}-(-y)\right\| \geqq\left\|x_{1}\right\|=\left\|-x_{1}\right\|$, which shows that $-x_{1} \in H_{\phi}^{1}$ and therefore $\alpha_{i} x_{1} \in H_{\phi}^{1}$ for $\alpha_{1} \in R$. Similarly, $\alpha_{2} x_{2} \in H_{\phi}^{2}$ for $\alpha_{2} \in R$. Thus, if we let $G=H^{1} \cap H^{2}, G_{\phi}$ is also a convex cone with vertex $\phi$ and hence contains $\alpha_{1} x_{1}+\alpha_{2} x_{2}$ for $\alpha_{1} \in R$ and $\alpha_{2} \in R$. Since $H^{1}$ and $H^{2}$ are hyperplanes, $E$ is the direct sum of $G$ and the two-dimensional sub-space $F$ spanned by $x_{1}$ and $x_{2}$. Therefore, if $z \in E, z=\left(\alpha_{1} x_{1}+\alpha_{2} x_{2}\right)-g$, where $-g \in G$, and $\alpha_{1} \in R, \alpha_{2} \in R$. Letting $f(z)=\alpha_{1} x_{1}+\alpha_{2} x_{2}$ we see that $f$ is a projection onto $F$ and since $f(z) \in G_{\phi}$ and $g \in G,\|f(z)\| \leqq\|f(z)-g\|=\|z\|$ and therefore $\|f\|=1$.

Thus, we can always find a projection of norm one on any twodimensional subspace of $E$ and therefore it is possible to define an inner product in any three-dimensional subspace of $E[9$, Theorem $3]$. This, however, implies that we can define an inner product in $E$ itself [8]. 
By a less direct argument than the above it can be shown that the conclusion still holds if the hypothesis " $S_{z}$ is convex whenever $z \in S$ and $S$ is convex" be replaced by " $L_{z}$ is convex whenever $L$ is a line and $z \in L . "$

Lemma 4.5. If $L_{z}$ is convex for each line $L$ and $z \in L$ then $E$ is strictly convex.

Proof. If $E$ is not strictly convex there exist distinct points $x$ and $y$ such that $\|\lambda x+(1-\lambda) y\|=1$ for each $\lambda \in[0,1]$. Let $L$ $=\{\lambda x+(1-\lambda) y: \lambda \in R\}$. If $\lambda>1,\|\lambda x+(1-\lambda) y\| \geqq \lambda\|x\|-|(1-\lambda)|\|y\|$ $=1$ while if $\lambda<0,\|\lambda x+(1-\lambda) y\| \geqq(1-\lambda)\|y\|-|\lambda|\|x\|=1$. Thus, $(1 / 2) x \in L_{(1 / 2)(x+y)}$ since if $z$ is any point of $L,\|z-(1 / 2) x\| \geqq\|z\|$ $-(1 / 2)\|x\| \geqq(1 / 2)=(1 / 2)\|y\|=\|(1 / 2)(x+y)-(1 / 2) x\|$. Similarly, $(1 / 2) y \in L_{(1 / 2)(x+y)}$. Further, $x+(1 / 2) y \in L_{(1 / 2)(x+y)}$, for if $z \in L$, then $x+y-z \in L$ and hence

$$
\begin{aligned}
\|(x+(1 / 2) y)-(1 / 2)(x+y)\| \\
\quad=\|(1 / 2) x\|=\|(1 / 2)(x+y)-(1 / 2) y\| \\
\leqq
\end{aligned}
$$

Since $L_{(1 / 2)(x+y)}$ is assumed to be convex,

$$
(1 / 2)[x+(1 / 2) y]+(1 / 2)[(1 / 2) x]=(3 / 4) x+(1 / 4) y \in L_{(1 / 2)(x+y)},
$$

which is impossible, $(3 / 4) x+(1 / 4) y$ itself being a point of $L$.

Lemma 4.6. Suppose $E$ is strictly convex and of dimension two. Then if $S$ is convex and $z \in S, S_{z}$ is a convex cone with vertex $z$.

Proof. By Lemma 3.1, $S_{z}$ is a cone with vertex $z$, so it remains only to show that $S_{z}$ is convex. Suppose $z=\phi$ and suppose $x \in S_{\phi}$ and $y \in S_{\phi}$; we must show that $[x, y] \subset S_{\phi}$.

Let $K$ be the closed convex cone generated by all the rays passing from $\phi$ through points of $[x, y]$. Then $K \cap S=\{\phi\}$, for if $w \in K \cap S$ there exists $\lambda \in] 0,1]$ such that $\lambda w$ is in the closed triangle $\phi x y$ and since $S$ is convex, $\lambda w \in S$. Let $u=(\|x\|+\|y\|)^{-1}(\|y\| x+\|x\| y)$. Then $u \in] x, y$ [ and hence $\lambda w$ is in the closed triangle $\phi u x$, say. (Otherwise $\lambda w$ is in $\phi u y$.) But if $\alpha u, \alpha \in[0,1]$, is any point of side $[\phi, u],\|x-\alpha u\|$ $\leqq\|x\|$. Consequently, $\|x-\lambda w\| \leqq\|x\|$ and so $x \in S_{\lambda w}$. By strict convexity and Lemma 3.2, $\lambda w=\phi$ and therefore $w=\phi$.

Now suppose $z \in[x, y]$ and $v \in S$. Then $[z, v]$ must intersect $\{\lambda x: \lambda \geqq 0\}$ or $\{\lambda y: \lambda \geqq 0\}$; say $\lambda x \in[z, v], \lambda \geqq 0$. Then $\lambda x \in S_{\phi}$ (since $S_{\phi}$ is a cone) and $\|z\| \leqq\|z-\lambda x\|+\|\lambda x\| \leqq\|z-\lambda x\|+\|\lambda x-v\|=\|z-v\|$. Since this holds for arbitrary $v \in S, z \in S_{\phi}$. 
5. The nearest-point map. If a closed set $S$ in $E$ is proximinal we can define a function $f$ from $E$ onto $S$ as follows: If $x \in E$ let $f(x)$ be a point of $S$ such that $x \in S_{f(x)}$. It is clear that $f$, called a nearest-point map for $S$, exists if and only if $S$ is proximinal, and that $f$ is unique if and only if $S$ is uniquely proximinal. We say that $f$ shrinks distances if $\|f(x)-f(y)\| \leqq\|x-y\|$ whenever $x, y \in E$. We will say that $E$ has the property $\mathrm{P}$ if a nearest-point map shrinks distances whenever it exists for a closed convex set $S \subset E$. The following theorem is well known, but a proof is included for completeness.

THEOREM 5.1. Each inner product space $E$ has the property P.

Proof. Suppose a nearest-point map $f$ exists for a closed convex set $S$. Since $E$ is strictly convex Lemma 3.2 implies that $f$ is unique. Suppose $x \in E$ and $y \in E$ and that $f(x)=\phi$. Let $H$ be the hyperplane through $\phi$ which is orthogonal to $f(y)$ and let $J$ be the open half-space determined by $H$ which contains $f(y)$. Let $K$ be the open half-space determined by $H+f(y)$ which contains $\phi$. If $x \in J$ there exists $\alpha>0$ such that $\|x\|>\|x-\alpha f(y)\|$. Pick $\lambda>0$ such that $\lambda \alpha=1 / 2$, then $\|\lambda x\|>\|\lambda x-(1 / 2) f(y)\|$. But, since $(1 / 2) f(y) \in S$, this contradicts the fact that $f(x)$, and hence $f(\lambda x)$, is the origin. We conclude that $x \notin J$ and an entirely similar argument shows that $y \notin K$. Thus, $\|x-y\|$ is no less than the width of $J \cap K$, and this is equal to $\|f(y)\|$.

Birkhoff [2] has defined a type of orthogonality which is meaningful in a general normed linear space $E$ and which coincides with the usual notion in an inner product space. If $x \neq 0$ we say that $y$ is orthogonal to $x$ (written $y \perp x$ ) if $\|y-\lambda x\| \geqq\|y\|$ for each $\lambda \in R$. Note that this is equivalent to saying that $y \in(R x)_{\phi}$, where $R x=\{\lambda x: \lambda \in R\}$ is the line determined by $x$ and $\phi$. We say that orthogonality is symmetric if $x \perp y$ implies $y \perp x$. Day [4, Theorem 6.4] and James [6, Theorem 1] have independently proved that a normed linear space of $d i$ mension at least three is an inner product space if and only if orthogonality is symmetric. We use this fact in proving the following theorem.

TheOREM 5.2. Suppose that the dimension of $E$ is at least three [resp. equal to two]. Then $E$ is an inner product space [resp. strictly convex and orthogonality is symmetric] if and only if $E$ has the property $\mathrm{P}$.

The proof is contained in Theorem 5.1 and the following succession of remarks and lemmas.

Lemma 5.3. If $E$ has the property $\mathrm{P}$ then $E$ is strictly convex.

Since the proof of this lemma is quite straightforward, it will be omitted. 
Lemma 5.4. If $E$ has the property $\mathrm{P}$ then orthogonality in $E$ is symmetric.

Proof. By Lemma 5.3, $E$ must be strictly convex and hence a nearest-point map is unique whenever it exists for a closed convex set. Suppose that neither $y$ nor $x$ is the origin and that $y \perp x$. The line $R x$ is uniquely proximinal and the nearest-point map $f$ exists for $R x$. Since $R y \subset(R x)_{\phi}, f(\lambda y)=\phi$ for any $\lambda \in R$. Now $E$ has the property P, so $\|x\|=\|f(x)-f(\lambda y)\| \leqq\|x-\lambda y\|$ for any $\lambda \in R$, i.e., $x \in(R y)_{\phi}$ or $x \perp y$. Thus, orthogonality is symmetric.

If the dimension of $E$ is at least three, the Day-James theorem mentioned above, together with Lemma 5.4, proves that if $E$ has property $\mathrm{P}$ it is an inner-product space.

Lemma 5.5. Suppose that $E$ is two-dimensional. If $E$ is strictly convex and orthogonality is symmetric then $E$ has the property $\mathrm{P}$.

Proof. Suppose the nearest-point map $f$ exists for a closed convex set $S$ and suppose $x, y \in E$. We can assume that $f(x)=\phi$. There exists a point $z \neq \phi$ such that $z \perp f(y)$ and, since $E$ is strictly convex, $w \perp f(y)$ implies $w \in R z$ [5, Theorem 4.3]. Let $J$ be the open half-space determined by $R z$ which contains $f(y)$ and let $K$ be the open half-space determined by $R z+f(y)$ which contains $\phi$. If $x \in J$ there exists a unique $\alpha \in R$ such that $x-\alpha f(y) \perp f(y)$ [5]. Now, $\alpha>0$ since $x-\alpha f(y)$ $\in R z$ and $x$ is on the same side of $R z$ as is $f(y)$. Thus, using strict convexity again, $\|x-\alpha f(y)\|<\|x\|$. As in the proof of Theorem 5.1 we conclude that $x \notin J$. A similar argument shows that $y \notin K$. Thus, $\|x-y\|$ is no less than the width of $K \cap J$. Now, by symmetry of orthogonality, $f(y) \perp z$ and so the distance from $f(y)$ to $R z$ is attained at $\phi$. Hence the distance from $R z+f(y)$ to $R z$ (which is the width of $J \cap K)$ is equal to $\|f(y)\|$ and therefore $\|f(y)\| \leqq\|x-y\|$.

It is not hard to see that neither strict convexity nor symmetry can be omitted in Lemma 5.5, since $\mathrm{P}$ implies both and there exist examples showing that neither implies the other.

The following theorem shows that the "shrinking" property of nearest-point maps is pretty well restricted to those which exist for convex sets.

Theorem 5.6. Suppose that $E$ is strictly convex and that a nearestpoint map $f$ exists for the closed set $S \subset E$. Then $S$ is convex if $f$ shrinks distances.

Proof. If $S$ is not convex there exist distinct points $x$ and $y$ of $S$ such that $] x, y[\subset E \sim S$. Letting $z=(1 / 2)(x+y)$ we see that one of 
$\|x-f(z)\|,\|y-f(z)\|$ is greater than (1/2)\|x-y\|. (This is obvious if $f(z)$ is $x$ or $y$, while if $f(z) \neq x, y$ and neither $\|x-f(z)\|$ nor $\|y-f(z)\|$ is greater than (1/2) $\|x-y\|$, strict convexity implies that $\|x-y\|$ $<\|x-f(z)\|+\|y-f(z)\| \leqq\|x-y\|$, a contradiction.) Suppose, then, that $\|x-f(z)\|>(1 / 2)\|x-y\|=\|x-z\|$. Since $f(x)=x$, this contradicts the assumption that $f$ shrinks distances. We get the same contradiction if $\|y-f(z)\|>(1 / 2)\|x-y\|$, hence $S$ must be convex.

A simple two-dimensional example can be constructed to show that we need to assume strict convexity in the above theorem.

Since every closed subset of Euclidean $n$-space $E^{n}$ is proximinal, Theorems 5.1 and 5.6 combine to give the following corollary.

CoRollary 5.7. Let $f$ be a nearest-point map for the closed set $S \subset E^{n}$. Then $S$ is convex if and only if $f$ shrinks distances.

\section{REFERENCES}

1. S. Banach, Theorie des operations linéaires, Warsaw, 1932.

2. G. Birkhoff, Orthogonality in linear metric spaces, Duke Math. J. vol. 1 (1935) pp. $169-172$.

3. H. Busemann, Note on a theorem of convex sets, Matematisk Tidsskrift B (1947) pp. 32-34.

4. M. M. Day, Some characterizations of inner-product spaces, Trans. Amer. Math. Soc. vol. 62 (1947) pp. 320-337.

5. R. C. James, Orthogonality and linear functionals, Trans. Amer. Math. Soc. vol. 61 (1947) pp. 265-292.

6. - Inner products in normed linear spaces, Bull. Amer. Math. Soc. vol. 53 (1947) pp. 559-566.

7. B. Jessen, Two theorems on convex point sets, Matematisk Tidsskrift. B (1940) pp. 66-70 (Danish).

8. P. Jordan and J. von Neumann, On inner products in linear spaces, Ann. of Math. (2) vol. 36 (1935) pp. 719-723.

9. S. Kakutani, Some characterizations of Euclidean space, Jap. J. Math. vol. 16 (1939) pp. 93-97.

10. V. L. Klee, A characterization of convex sets, Amer. Math. Monthly vol. 56 (1949) pp. 247-249.

11. Th. Motzkin, Sur quelques propriettes caractéristiques des ensembles convexes, Atti Acad. Naz. Lincei Rend 6 vol. 21 (1935) pp. 562-567.

12. - Sur quelques proprietés caractéristiques des ensembles bornés non convexes, Atti Acad. Naz. Lincei Rend 6 vol. 21 (1935) pp. 773-779.

University of California, Los Angeles and UNIVERSITY OF WASHINGTON 